\title{
The free carboxylate stretching modes
}

\author{
Jos Oomens*, Jeffrey D. Steill \\ FOM Institute for Plasma Physics “Rijnhuizen, Nieuwegein, The Netherlands
}

\section{SUPPORTING INFORMATION}

Optimized structures (Angstrom) with un-scaled harmonic vibrational frequencies $\left(\mathrm{cm}^{-1}\right)$ and infrared absorption intensity $(\mathrm{km} / \mathrm{mol})$ values calculated at the B3LYP/aug-cc-pVDZ level of theory:

Benzoate Anion

B3LYP/aug-cc-pVDZ

Structure

\begin{tabular}{|c|c|c|c|}
\hline Atomic number & $\mathbf{X}$ & $\mathbf{Y}$ & $\mathbf{Z}$ \\
\hline 6 & 0 & 1.2095 & 1.837101 \\
\hline 6 & 0 & 1.2053 & 0.437246 \\
\hline 6 & 0 & 0.0000 & -0.281675 \\
\hline 6 & 0 & -1.2053 & 0.437246 \\
\hline 6 & 0 & -1.2095 & 1.837101 \\
\hline 6 & 0 & 0.0000 & 2.544626 \\
\hline 1 & 0 & 2.1577 & 2.381602 \\
\hline 1 & 0 & 2.1316 & -0.138285 \\
\hline 1 & 0 & -2.1316 & -0.138285 \\
\hline 1 & 0 & -2.1577 & 2.381602 \\
\hline 1 & 0 & 0.0000 & 3.637419 \\
\hline 6 & 0 & 0.0000 & -1.833346 \\
\hline 8 & 0 & -1.1369 & -2.374615 \\
\hline 8 & 0 & 1.1369 & -2.374615 \\
\hline
\end{tabular}

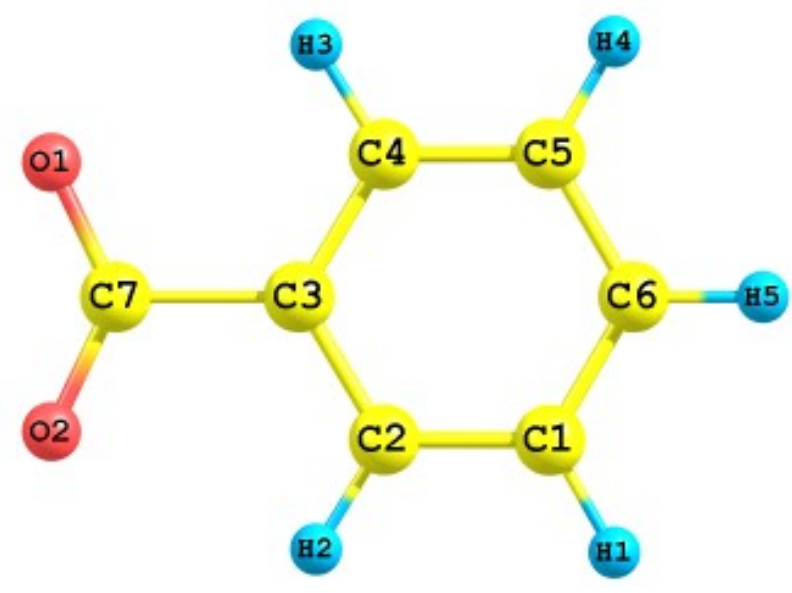

\begin{tabular}{l} 
Vibrations \\
\begin{tabular}{|c|c|}
\hline Frequency & Intensity \\
\hline 62 & 0.0 \\
\hline 163 & 3.1 \\
\hline 215 & 0.3 \\
\hline 366 & 8.7 \\
\hline 425 & 0.0 \\
\hline 457 & 0.1 \\
\hline 500 & 4.1 \\
\hline 626 & 0.1 \\
\hline 672 & 15.5 \\
\hline 706 & 60.0 \\
\hline 713 & 39.4 \\
\hline 806 & 51.8 \\
\hline 829 & 0.0 \\
\hline 852 & 0.0 \\
\hline 938 & 2.0 \\
\hline 992 & 0.8 \\
\hline 993 & 0.0 \\
\hline 1004 & 2.0 \\
\hline 1035 & 17.3 \\
\hline 1065 & 9.6 \\
\hline 1118 & 5.8 \\
\hline 1153 & 0.1 \\
\hline 1165 & 4.7 \\
\hline 1295 & 0.0 \\
\hline 1338 & 320.8 \\
\hline 1350 & 1.9 \\
\hline 1449 & 0.0 \\
\hline
\end{tabular} \\
\hline
\end{tabular}




\begin{tabular}{|c|c|}
1486 & 5.8 \\
\hline 1606 & 61.9 \\
\hline 1624 & 2.5 \\
\hline 1659 & 425.4 \\
\hline 3131 & 0.7 \\
\hline 3142 & 55.4 \\
\hline 3162 & 64.6 \\
\hline 3189 & 17.7 \\
\hline 3190 & 9.1795 \\
\hline
\end{tabular}

Benzoate Anion B3LYP/6-31+G(d,p)

Structure

\begin{tabular}{|c|c|c|c|}
\hline Atomic number & $\mathbf{X}$ & $\mathbf{Y}$ & $\mathbf{Z}$ \\
\hline 6 & 0 & -1.208651 & 1.836177 \\
\hline 6 & 0 & -1.204418 & 0.437524 \\
\hline 6 & 0 & 0.000000 & -0.280560 \\
\hline 6 & 0 & 1.204418 & 0.437524 \\
\hline 6 & 0 & 1.208651 & 1.836177 \\
\hline 6 & 0 & 0.000000 & 2.543254 \\
\hline 1 & 0 & -2.153339 & 2.377892 \\
\hline 1 & 0 & -2.126206 & -0.136918 \\
\hline 1 & 0 & 2.126206 & -0.136918 \\
\hline 1 & 0 & 2.153339 & 2.377892 \\
\hline 1 & 0 & 0.000000 & 3.631654 \\
\hline 6 & 0 & 0.000000 & -1.832843 \\
\hline 8 & 0 & 1.137968 & -2.373570 \\
\hline 8 & 0 & -1.137968 & -2.373570 \\
\hline
\end{tabular}

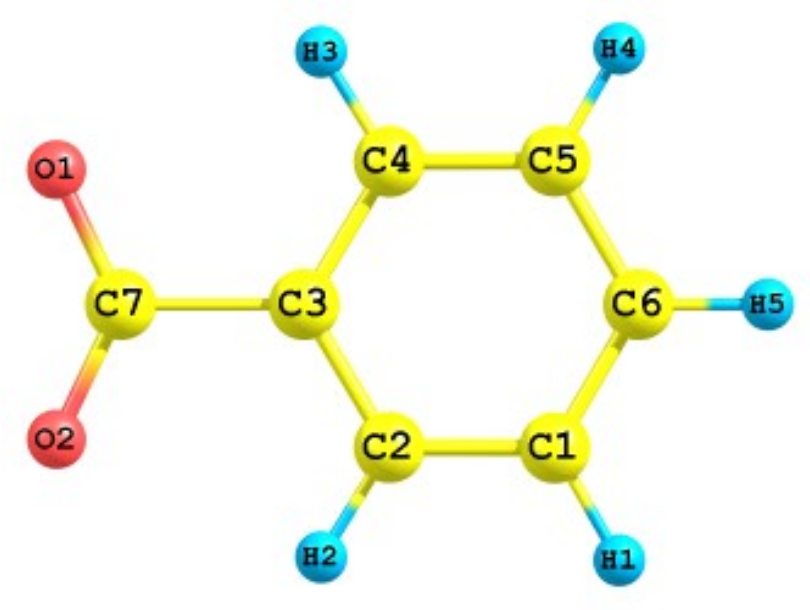

Vibrations

\begin{tabular}{|c|c|}
\hline Frequency & Intensity \\
\hline 63 & 0 \\
\hline 159 & 3.2 \\
\hline 214 & 0.4 \\
\hline 366 & 8.0 \\
\hline 423 & 0 \\
\hline 445 & 0.1 \\
\hline 500 & 3.8 \\
\hline 630 & 0.1 \\
\hline 675 & 17.0 \\
\hline 688 & 0.5 \\
\hline 714 & 108.6 \\
\hline 809 & 57.0 \\
\hline 814 & 1.2 \\
\hline 858 & 0 \\
\hline 936 & 2.6 \\
\hline 988 & 1.4 \\
\hline 995 & 0 \\
\hline 1012 & 2.3 \\
\hline 1041 & 14.8 \\
\hline 1074 & 9.7 \\
\hline 1125 & 7.5 \\
\hline 1167 & 0.1 \\
\hline 1177 & 4.3 \\
\hline 1314 & 0.0 \\
\hline 1347 & 339.8 \\
\hline 1352 & 1.8 \\
\hline 1469 & 0.0 \\
\hline 1506 & 5.7 \\
\hline 1615 & 41.7 \\
\hline 1632 & 2.3 \\
\hline 1681 & 458.1 \\
\hline 3139 & 0.6 \\
\hline 3150 & 61.5 \\
\hline 3170 & 72.5 \\
\hline 3199 & 17.7 \\
\hline
\end{tabular}


Para-fluorobenzoate Anion

B3LYP/aug-cc-pVDZ

Structure

\begin{tabular}{|c|c|c|c|}
\hline Atomic number & $\mathbf{X}$ & $\mathbf{Y}$ & $\mathbf{Z}$ \\
\hline 6 & 0 & 0.0000 & -2.272331 \\
\hline 6 & 0 & 0.0000 & -0.721396 \\
\hline 6 & 0 & 1.2043 & -0.002893 \\
\hline 6 & 0 & 1.2189 & 1.398108 \\
\hline 6 & 0 & -1.2189 & 1.398108 \\
\hline 6 & 0 & -1.2043 & -0.002893 \\
\hline 1 & 0 & 2.1314 & -0.576548 \\
\hline 1 & 0 & 2.1500 & 1.966588 \\
\hline 1 & 0 & -2.1500 & 1.966588 \\
\hline 1 & 0 & -2.1314 & -0.576548 \\
\hline 6 & 0 & 0.0000 & 2.066454 \\
\hline 8 & 0 & 1.1375 & -2.81132 \\
\hline 8 & 0 & -1.1375 & -2.81132 \\
\hline 9 & 0 & 0.0000 & 3.446898 \\
\hline
\end{tabular}

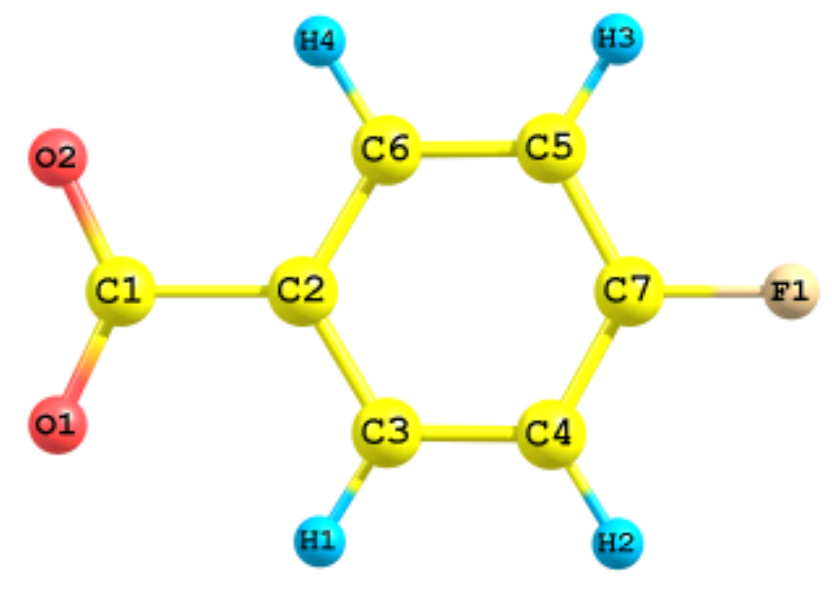

Vibrations

\begin{tabular}{|c|c|}
\hline Frequency & Intensity \\
\hline 66 & 0.0 \\
\hline 111 & 7.0 \\
\hline 192 & 1.8 \\
\hline 308 & 0.7 \\
\hline 333 & 3.5 \\
\hline 389 & 4.1 \\
\hline 435 & 0.0 \\
\hline 499 & 7.0 \\
\hline 511 & 2.7 \\
\hline 597 & 43.7 \\
\hline 638 & 0.1 \\
\hline 696 & 17.2 \\
\hline 771 & 2.9 \\
\hline 791 & 45.1 \\
\hline 812 & 0.0 \\
\hline 835 & 99.9 \\
\hline 865 & 20.3 \\
\hline 976 & 0.6 \\
\hline 989 & 0.0 \\
\hline 1022 & 12.3 \\
\hline 1077 & 11.5 \\
\hline 1119 & 4.3 \\
\hline 1142 & 31.8 \\
\hline 1191 & 94.7 \\
\hline 1270 & 1.4 \\
\hline 1337 & 313.6 \\
\hline 1349 & 3.2 \\
\hline 1409 & 1.5 \\
\hline 1499 & 81.9 \\
\hline 1621 & 32.0 \\
\hline 1625 & 59.6 \\
\hline 1666 & 431.4 \\
\hline 3174 & 16.8 \\
\hline 3175 & 12.7 \\
\hline 3196 & 13.0 \\
\hline 3197 & 11.9 \\
\hline
\end{tabular}


Meta-fluorobenzoate Anion B3LYP/aug-cc-pVDZ

Structure

\begin{tabular}{|c|c|c|c|}
\hline Atomic number & $\mathbf{X}$ & $\mathbf{Y}$ & $\mathbf{Z}$ \\
\hline 6 & -0.678092 & 2.0242 & 0 \\
\hline 6 & 0 & 0.6258 & 0 \\
\hline 6 & -0.785195 & -0.5375 & 0 \\
\hline 6 & -0.155799 & -1.7741 & 0 \\
\hline 6 & 2.005661 & -0.7585 & 0 \\
\hline 6 & 1.397892 & 0.5010 & 0 \\
\hline 1 & -1.870109 & -0.4399 & 0 \\
\hline 1 & 3.095094 & -0.8435 & 0 \\
\hline 1 & 1.983596 & 1.4198 & 0 \\
\hline 6 & 1.227327 & -1.9249 & 0 \\
\hline 8 & -1.935825 & 2.0111 & 0 \\
\hline 8 & 0.112007 & 3.0031 & 0 \\
\hline 1 & 1.671144 & -2.9206 & 0 \\
\hline 9 & -0.928883 & -2.9183 & 0 \\
\hline
\end{tabular}

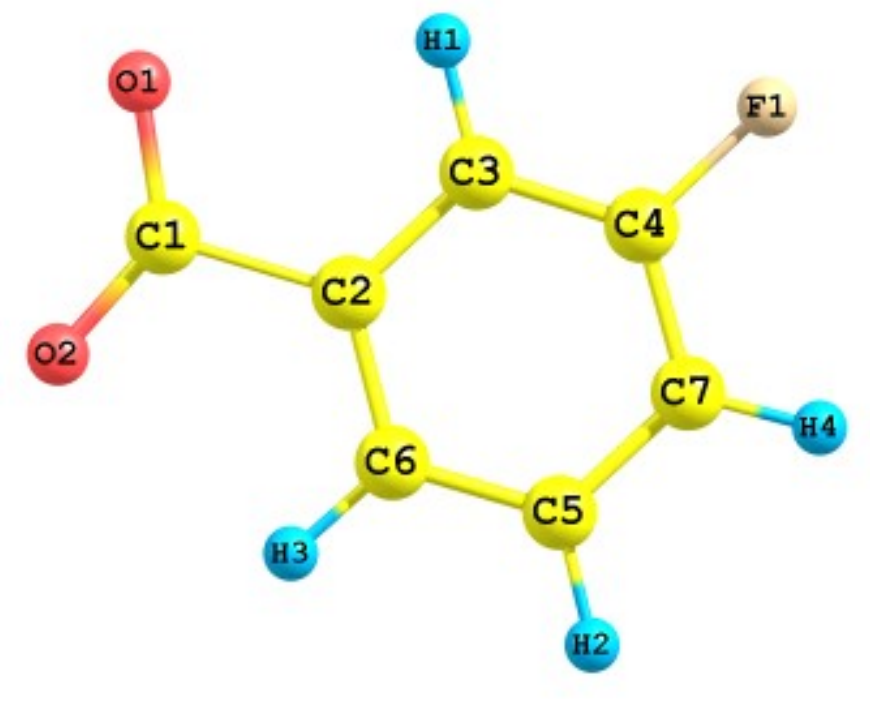

Vibrations

\begin{tabular}{|c|c|}
\hline Frequency & Intensity \\
\hline 55 & 0.3 \\
\hline 157 & 4.0 \\
\hline 185 & 1.5 \\
\hline 245 & 0.0 \\
\hline 347 & 4.8 \\
\hline 394 & 7.6 \\
\hline 453 & 0.4 \\
\hline 494 & 1.2 \\
\hline 540 & 7.6 \\
\hline 542 & 10.8 \\
\hline 667 & 4.6 \\
\hline 695 & 7.0 \\
\hline 767 & 66.8 \\
\hline 770 & 95.4 \\
\hline 814 & 0.2 \\
\hline 899 & 34.0 \\
\hline 904 & 0.4 \\
\hline 934 & 12.0 \\
\hline 985 & 0.5 \\
\hline 1009 & 3.0 \\
\hline 1063 & 0.2 \\
\hline 1078 & 24.7 \\
\hline 1147 & 0.9 \\
\hline 1198 & 97.3 \\
\hline 1256 & 14.1 \\
\hline 1332 & 328.9 \\
\hline 1354 & 1.0 \\
\hline 1441 & 14.5 \\
\hline 1478 & 33.7 \\
\hline 1609 & 148.6 \\
\hline 1637 & 37.0 \\
\hline 1666 & 388.5 \\
\hline 3149 & 30.3 \\
\hline 3189 & 19.0 \\
\hline 3199 & 12.1 \\
\hline 3205 & 8.4 \\
\hline
\end{tabular}


Ortho-fluorobenzoate

Anion

B3LYP/aug-cc-pVDZ

Structure

\begin{tabular}{|c|c|c|c|}
\hline Atomic number & $\mathbf{X}$ & $\mathbf{Y}$ & $\mathbf{Z}$ \\
\hline 6 & -1.826912 & -0.2897 & 0.031903 \\
\hline 6 & -0.279503 & -0.2465 & 0.009632 \\
\hline 6 & 0.474746 & 0.9243 & -0.099281 \\
\hline 6 & 1.868451 & 0.9486 & -0.090594 \\
\hline 6 & 1.853912 & -1.4535 & 0.163037 \\
\hline 6 & 0.456228 & -1.4389 & 0.124411 \\
\hline 1 & 2.387621 & -2.4006 & 0.268065 \\
\hline 1 & -0.106632 & -2.3721 & 0.171496 \\
\hline 6 & 2.568139 & -0.2544 & 0.05297 \\
\hline 8 & -2.363776 & 0.4127 & 0.920407 \\
\hline 8 & -2.338642 & -1.0587 & -0.819887 \\
\hline 1 & 3.659605 & -0.2512 & 0.069454 \\
\hline 1 & 2.385985 & 1.9029 & -0.196406 \\
\hline 9 & -0.155289 & 2.1278 & -0.252138 \\
\hline
\end{tabular}

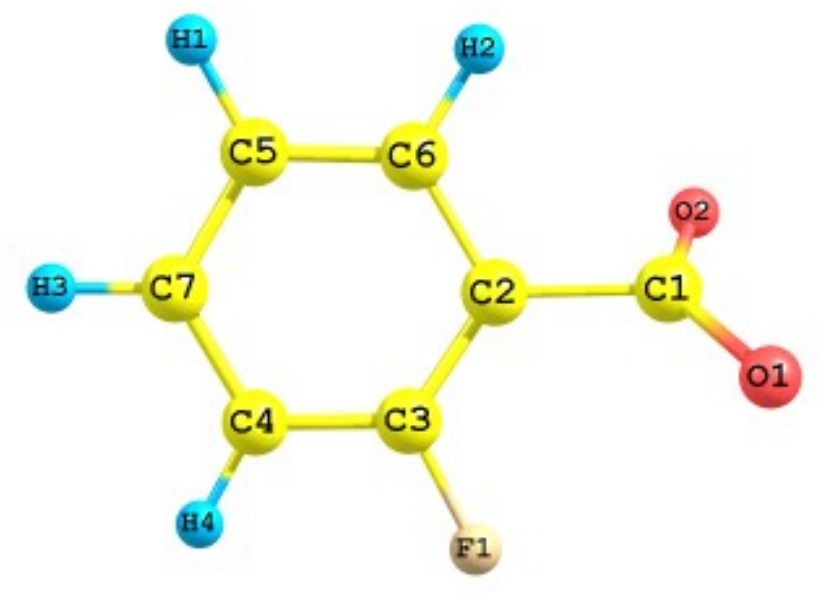

Vibrations

\begin{tabular}{|c|c|}
\hline Frequency & Intensity \\
\hline 20 & 0.4 \\
\hline 115 & 0.9 \\
\hline 167 & 2.9 \\
\hline 229 & 0.7 \\
\hline 314 & 4.5 \\
\hline 368 & 1.1 \\
\hline 449 & 4.3 \\
\hline 495 & 4.8 \\
\hline 541 & 7.8 \\
\hline 589 & 0.8 \\
\hline 653 & 17.3 \\
\hline 725 & 25.6 \\
\hline 748 & 54.8 \\
\hline 777 & 33.8 \\
\hline 797 & 14.7 \\
\hline 838 & 27.1 \\
\hline 857 & 0.5 \\
\hline 927 & 3.3 \\
\hline 960 & 0.9 \\
\hline 1046 & 11.8 \\
\hline 1091 & 19.5 \\
\hline 1137 & 0.6 \\
\hline 1157 & 0.1 \\
\hline 1210 & 79.9 \\
\hline 1260 & 0.9 \\
\hline 1339 & 332.0 \\
\hline 1349 & 4.6 \\
\hline 1449 & 17.7 \\
\hline 1485 & 51.2 \\
\hline 1605 & 15.9 \\
\hline 1629 & 1.2 \\
\hline 1669 & 576.6 \\
\hline 3149 & 7.1 \\
\hline 3167 & 32.1 \\
\hline 3183 & 32.7 \\
\hline 3187 & 21.6 \\
\hline
\end{tabular}


Para-aminobenzoate Anion B3LYPlaug-cc-pVDZ

Structure

\begin{tabular}{|c|c|c|c|}
\hline Atomic number & $\mathbf{X}$ & $\mathbf{Y}$ & $\mathbf{Z}$ \\
\hline 6 & 0.000583 & 2.2994 & 0 \\
\hline 6 & -0.00676 & 0.7521 & 0 \\
\hline 6 & -0.008662 & 0.0251 & 1.198695 \\
\hline 6 & -0.008662 & -1.3735 & 1.206725 \\
\hline 6 & -0.008662 & -1.3735 & -1.206725 \\
\hline 6 & -0.008662 & 0.0251 & -1.198695 \\
\hline 1 & -0.00678 & 0.5932 & 2.129471 \\
\hline 1 & -0.014237 & -1.9185 & 2.155725 \\
\hline 1 & -0.014237 & -1.9185 & -2.155725 \\
\hline 1 & -0.00678 & 0.5932 & -2.129471 \\
\hline 6 & -0.00666 & -2.0902 & 0 \\
\hline 8 & 0.00387 & 2.8428 & 1.136922 \\
\hline 8 & 0.00387 & 2.8428 & -1.136922 \\
\hline 7 & -0.063686 & -3.5117 & 0 \\
\hline 1 & 0.355413 & -3.9191 & -0.828574 \\
\hline 1 & 0.355413 & -3.9191 & 0.828574 \\
\hline
\end{tabular}

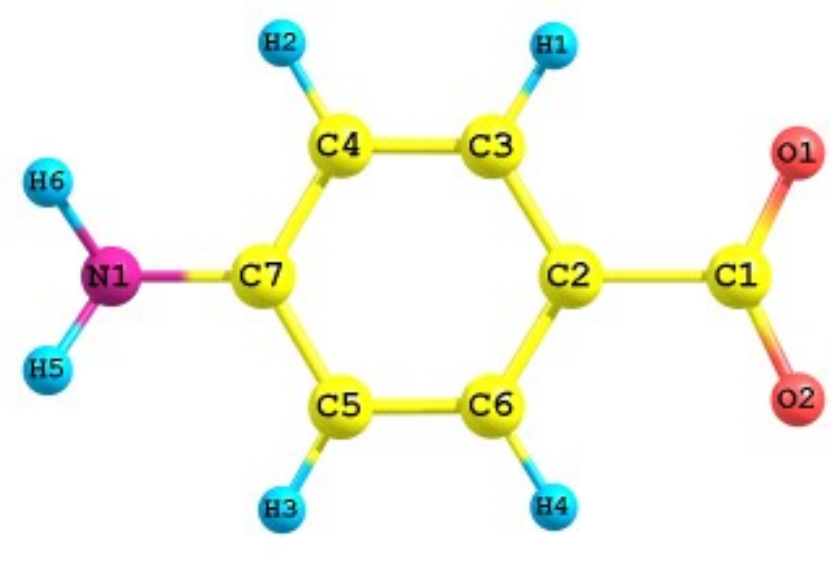

Vibrations

\begin{tabular}{|c|c|}
\hline Frequency & Intensity \\
\hline 67 & 0.3 \\
\hline 108 & 2.3 \\
\hline 188 & 2.8 \\
\hline 194 & 19.6 \\
\hline 292 & 10.3 \\
\hline 338 & 6.9 \\
\hline 374 & 0.6 \\
\hline 427 & 0.2 \\
\hline 501 & 32.9 \\
\hline 511 & 4.3 \\
\hline 611 & 57.2 \\
\hline 646 & 0.2 \\
\hline 681 & 156.6 \\
\hline 710 & 43.5 \\
\hline 778 & 22.5 \\
\hline 797 & 104.9 \\
\hline 811 & 0.0 \\
\hline 841 & 33.6 \\
\hline 856 & 23.4 \\
\hline 974 & 1.2 \\
\hline 990 & 0.0 \\
\hline 1019 & 4.0 \\
\hline 1071 & 11.6 \\
\hline 1122 & 14.2 \\
\hline 1124 & 2.4 \\
\hline 1170 & 19.1 \\
\hline 1255 & 79.6 \\
\hline 1295 & 0.0 \\
\hline 1339 & 362.1 \\
\hline 1354 & 0.1 \\
\hline 1441 & 0.6 \\
\hline 1513 & 51.3 \\
\hline 1611 & 65.4 \\
\hline 1622 & 16.9 \\
\hline 1641 & 87.7 \\
\hline 1656 & 431.3 \\
\hline 3126 & 51.0 \\
\hline 3128 & 46.8 \\
\hline 3189 & 14.1 \\
\hline 3190 & 4.3 \\
\hline 3520 & 0.1 \\
\hline 3618 & 3.4 \\
\hline
\end{tabular}


Comparison of harmonic and anharmonic vibrational frequencies for the benzoate anion at the B3LYP/aug-cc-pVDZ level of theory.

Harmonic vs. Anharmonic Frequency Calculations

\begin{tabular}{|c|c|c|c|}
\hline $\begin{array}{l}\text { Harmonic } \\
\text { Frequencies }\end{array}$ & $\begin{array}{l}\text { Anharmonic } \\
\text { Frequencies }\end{array}$ & $\Delta$ & $\Delta /($ harmonic) \\
\hline 62 & 53 & 8.6 & 0.14 \\
\hline 163 & 151 & 12.0 & 0.07 \\
\hline 215 & 212 & 3.2 & 0.02 \\
\hline 366 & 362 & 4.0 & 0.01 \\
\hline 425 & 405 & 19.8 & 0.05 \\
\hline 457 & 429 & 27.3 & 0.06 \\
\hline 500 & 483 & 16.4 & 0.03 \\
\hline 626 & 625 & 0.7 & 0.00 \\
\hline 672 & 668 & 4.4 & 0.01 \\
\hline 706 & 679 & 27.5 & 0.04 \\
\hline 713 & 660 & 53.3 & 0.07 \\
\hline 806 & 797 & 8.8 & 0.01 \\
\hline 829 & 793 & 35.8 & 0.04 \\
\hline 852 & 824 & 28.2 & 0.03 \\
\hline 938 & 888 & 50.2 & 0.05 \\
\hline 992 & 903 & 89.6 & 0.09 \\
\hline 993 & 945 & 47.6 & 0.05 \\
\hline 1004 & 1008 & -4.3 & 0.00 \\
\hline 1035 & 1019 & 15.2 & 0.01 \\
\hline 1065 & 1048 & 16.9 & 0.02 \\
\hline 1118 & 1101 & 17.4 & 0.02 \\
\hline 1153 & 1145 & 8.1 & 0.01 \\
\hline 1165 & 1151 & 13.4 & 0.01 \\
\hline 1295 & 1289 & 6.4 & 0.00 \\
\hline 1338 & 1307 & 30.3 & 0.02 \\
\hline 1350 & 1321 & 29.0 & 0.02 \\
\hline 1449 & 1455 & -5.6 & 0.00 \\
\hline 1486 & 1484 & 1.9 & 0.00 \\
\hline 1606 & 1568 & 38.0 & 0.02 \\
\hline 1624 & 1587 & 37.2 & 0.02 \\
\hline 1659 & 1629 & 30.4 & 0.02 \\
\hline 3131 & 3012 & 119.0 & 0.04 \\
\hline 3142 & 2997 & 144.3 & 0.05 \\
\hline 3162 & 3017 & 145.4 & 0.05 \\
\hline 3189 & 3043 & 146.6 & 0.05 \\
\hline 3190 & 3037 & 152.6 & 0.05 \\
\hline
\end{tabular}

